\title{
Effective Conductivity of Graphene Quantum Dots Solution
}

\author{
Ruxia CHEN ${ }^{1, \mathrm{a}}$, Yufeng PENG ${ }^{1, \mathrm{~b}, *}$, Mingjie $\mathrm{LI}^{1, \mathrm{c}}$, Xueyun HAN ${ }^{1, \mathrm{~d}}$ \\ ${ }^{1}$ College of Physics and Materials Science \\ Henan Normal University, \\ Xinxiang, China \\ e-mail: ${ }^{\mathrm{a} C h e n r u x i a 1992 @ 163 . c o m}{ }^{1}$, byufengp@ @tu.cn, ${ }^{\mathrm{c}}$ mingjie8699@126.com, ${ }^{\mathrm{d}}$ hanxueyun126@126.com
}

\begin{abstract}
In this paper, a composite material model with ellipsoidal conductive particles was used to establish an effective conductivity model for studying graphene quantum dots (GQDs) solution. By comparing effective media theoretical (EMT) model, porous flow theory model and the general effective model (GEM), we finally selected the general effective model (GEM) to fit the concentration dependence of the effective conductivity of the GQDs. This model perfectly explained our experimental data. The GEM model is able to predict the effective conductivity of GQDs solution. On the other hand, we modified the tunnel conductivity model based on the analysis about the tunnel conductivity model and Arrhenius equation. The modified model well simulated the temperature dependence of the effective conductivity of GQDs solution and provided a more reliable guidance for the design and optimization of the GQDs composites.
\end{abstract}

Keywords-GQDs; effective conductivity model; EMT; GEM, tunnel conductivity model

\section{INTRODUCTION}

In recent years, GQDs have been studied extensively, because their electronic properties can be tuned by controlling the size and the functional groups of GQDs. Taking advantage of the electrochemical properties similar to graphene, GQDs are widely used as a kind of suitable electrode materials, not only in supercapacitors [1], photovoltaic cells [2] or solar cells [3], but also in the field of sensors [4]. Notably, due to the quantum confinement and edge effects, GQDs exhibit high Photoluminescence and slow hot-carrier relaxation [4-5]. With these advantages, GQDs have better performance comparing to conventional semiconductor in Qcatalytic reactions [6], conductive material [7], detection ions [8], probes [9] and bioimaging [10]. GQDs with heteroatom doping are able to improve some properties of materials or instruments [11]. The previous experiments showed that the GQDs didn't have obvious toxicity to the organism [12].

At present, there many study of conductivity about the graphene and its composite material. But many fundamental theoretical problems of the conductivity are still not resolved, which encourages many scientists to work on them.[13]. Furthermore, there are no theoretical models and experiments on the conductivity of GQDs solutions. In this work, we studied the concentration, temperature dependence of the effective conductivity of GQDs by different composite models, and presented the formula for low density approximation.

\section{THEORY}

\section{A General Effective Media (GEM) model}

According to the theory of steady electric field, an ellipsoidal particle with the conductivity as $\sigma \mathrm{m}$ was suspended on the substrate phase with the conductivity as $\sigma \mathrm{n}$. Moreover, $\mathrm{c} 1$ was the volume concentration of conductive particles. The coordinates of the ellipsoid were measured using Cartesian coordinate system (X, Y, Z). When ni=1/3, use self-consistent conditions for the system, the formula of the EMT is able to obtain

$$
c_{1}\left(\frac{\sigma_{m}-\sigma}{2 \sigma+\sigma_{m}}\right)+\left(1-c_{1}\right) \frac{\left(\sigma_{n}-\sigma\right)}{2 \sigma+\sigma_{n}}=0
$$

By the porous flow theory, if the conductive particles contact with each other or the particle gap is within $1 \mathrm{~nm}$, the composites can be considered that the formation of conductive channels. The equation of the effective conductivity of the conductive composites near the percolation threshold can be written as:

$$
\sigma^{1 / f}=\sigma_{m}^{1 / f}\left(\kappa_{m}-\kappa *\right)
$$

Where $\kappa^{*}$ is the percolation threshold; $\mathrm{f}$ is the key index of the system. The value of $t$ depends on the dimension of the material and on the size and morphology of the conductive filler. Usually, the value of $t$ is obtained through the computer simulation. The value of $f$ in the actual system usually exceeds its limit from the above theory because of the influence of dimension and morphology of the conductive filler system. McLachlan has proposed a more universal model (General Effective Media (GEM) model). GEM model combines seepage theory and effective medium theory. The general form is shown as follows:

$$
\frac{c_{m}\left(\sigma_{1}^{1 / f}-\sigma^{1 / f}\right)}{\sigma_{m}^{1 / f}+\sigma_{m}^{1 / f}}+\frac{\left(1-c_{m}\right)\left(\sigma_{n}^{1 / f}-\sigma^{1 / f}\right)}{\sigma_{n}^{1 / t}+\sigma^{1 / f}}=0
$$

The conductivity of the conductive particles is much larger than that of the insulating substrate. Therefore, 
$\left(\sigma_{n} / \sigma_{m}\right)^{1 / f}$ is approximately equal to 0 . The GEM model can be simplified as:

$$
\sigma=\sigma_{m}\left[\kappa_{m}-\frac{\left(1-\kappa_{m}\right) \kappa^{*}}{1-\kappa^{*}}\right]^{f}
$$

\section{$B$ Temperature dependence of electrolyte conductance}

The composite will be conductive if the composite substrate is an insulating material. The filler material (particles, powder or poop etc.) is a conductive material with certain content and uniformly dispersed in the substrate. Obviously, the conductivities of these composites mainly depend on the ability of electrons to cross the barrier. The barrier is related to not only the thickness of the base layer between the particles, but also the nature of the substrate. According to tunneling theory available,

$$
\sigma=\sigma_{m} \exp \left(\frac{-T_{1}}{T+T_{2}}\right)
$$

$\mathrm{T}_{1}$ and $\mathrm{T}_{2}$ are two constants. The above equation shows that the conductivities of these composites are depended largely on the temperature. If it is difficult for the electrons to break through the barrier material constituting the insulation, then $T$ is much bigger than $T_{n}\left(T \gg T_{n}\right)$ and the equation (5) can be written as follow:

$$
\sigma=\sigma_{m} \exp \left(\frac{-Q}{K_{B} T}\right)
$$

Q is the activation energy of the electron through the barrier. It can be seen from the formula that the electrical conductivity of the composite increases with the temperature. The general form of the Arrhenius equation is as below:

$$
k=k_{0} T^{\alpha} \exp \left(-\frac{Q}{R T}\right)
$$

Where $\alpha$ is an additional parameter that is estimated from the available experimental data. It is usually within the range of $(0,1)$, and occasionally greater than 1 . The temperature dependency of conductivity can be expressed as a simplified Arrhenius equation:

$$
\sigma=\frac{\sigma_{0}}{T} \exp \left(-\frac{Q}{K_{B} T}\right)
$$

$\sigma_{0}$ is the pre-exponential factor of the conductivity. It is a constant only determined by the nature of the reaction, and it has nothing to do with the reaction temperature and the concentration of the substance in the system. Drawing on formulas (6), (7) and (8), the relationship between the effective conductivity and the temperature can be corrected to:

$$
\sigma=\sigma_{m} T^{\alpha} \exp \left(\frac{-Q}{K_{B} T}\right)
$$

Taking $\log$ the expression gives,

$$
\ln \sigma=\ln \sigma_{m}-\alpha \ln 1 / T-\frac{Q}{K_{B} T}
$$

Where $\alpha$ is index factor for the $\mathrm{T}$.

\section{EXPERIMENT}

\section{A Preparation of the experimental solution}

Graphene quantum dots (2g/L) purchased from QINGDAO HAIDA HAIXI NEW MATERLALS CO., LTD. Secondary distilled water prepared from the laboratory was used directly without any further treatment. The solutions were prepared by weighing (the balance, model WT603CH) with an accuracy of $\pm 1 \times 10^{-3} \mathrm{~g}$. The uncertainty of the concentration is $\pm 2 \times 10^{-3}$.

\section{B Apparatus}

The conductivity of GQDs was measured by Microprocessor conductivity meter (DDS-W). The temperature of GQDs was recorded by Dual input $\mathrm{K}$ thermometer (TA8112). The temperature changes by using Phase shift trigger temperature control box (5188). Add the magnetic field by using Electromagnet (PEM-30). The magnetic field was measured by Gauss meter (HT201). The conductivity of GQDs was recorded by LCR-819. The frequency of GQDs was measured by Network analyzer. The transmission electron microscopy (TEM) image of GQDs was taken by using a Tecnai-G2 F20. The measurement accuracy of instruments can be seen in table 1 .

\section{Characterization}

As shown in Fig.1, the sizes of GQDs were investigated by Transmission Electron Microscope (TEM). The particles were evenly distributed in the matrix. The particle size distribution (PSD) histogram was obtained by choosing 100 particles in TEM image. The GQDs had an average particle size of $11 \mathrm{~nm}$ which was measured by Dynamic Light Scattering (DLS; Fig.2a). As shown in Fig.2 (b), the GQDs consisted as the graphene fragments within $0.4-1.8 \mathrm{~nm}$ in height, and the corresponding graphene layers were 1-3 layers. 
TABLE I. THE INSTRUMENT USED IN THE EXPERIMENT

\begin{tabular}{|c|c|c|}
\hline Equipment name & Measurement accuracy & Manufacturer \\
\hline Microprocessor conductivity meter(DDS-W) & $0.2 \%$ & BANTE \\
\hline Phase shift trigger temperature control box(5188) & $\pm 0.2^{\circ} \mathrm{C}$ & TASI \\
\hline Dual input K thermometer(TA8112) & $\pm 0.1^{\circ} \mathrm{C}$ & HUATAI \\
\hline DC power supply(HCP06-60) & Simplex220V $\pm 10 \%$ & LITIAN \\
\hline Electromagnet(PEM-30) & Hesic Error: $\pm 2 \%$ & GW \\
\hline Gauss meter(HT201) & $0.05 \%$ & FEI \\
\hline LCR-819 & cs: $1.2 \mathrm{~nm}$ & Agilent \\
\hline Tecnai-G2 F20 & $\pm 1.1 \mathrm{db}$ & \\
\hline Network analyzer & & \\
\hline
\end{tabular}

(a)

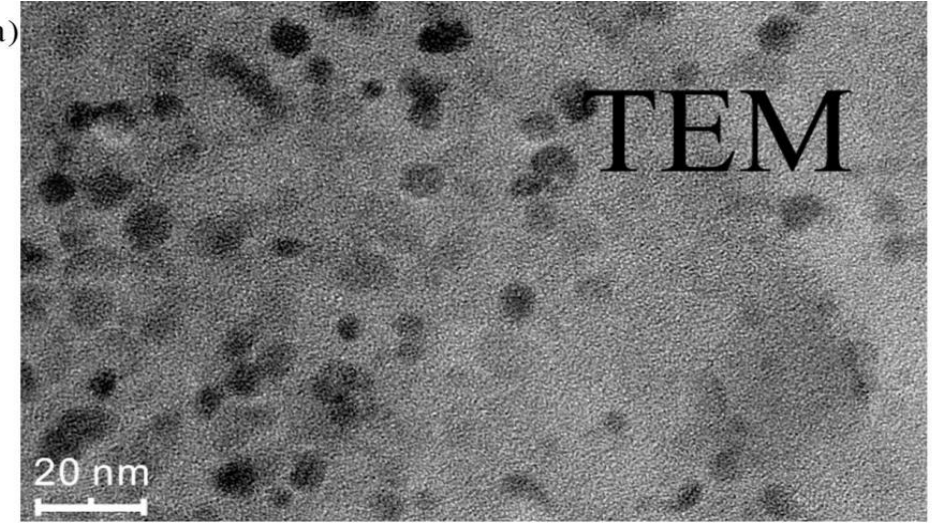

(b)

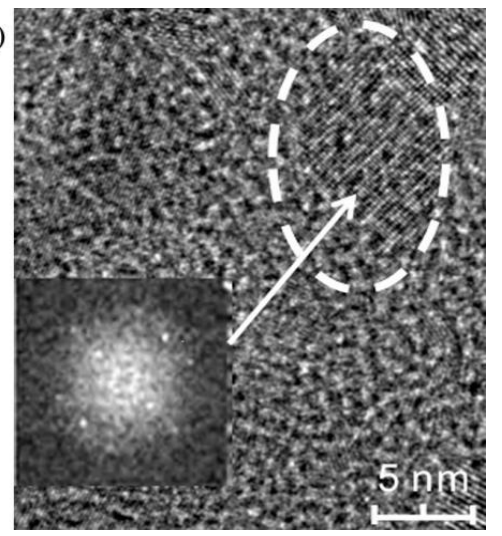

Figure1. (a) TEM image. (b) High times TEM image. Interpolation diagram is the Fourier transform diffraction pattern.
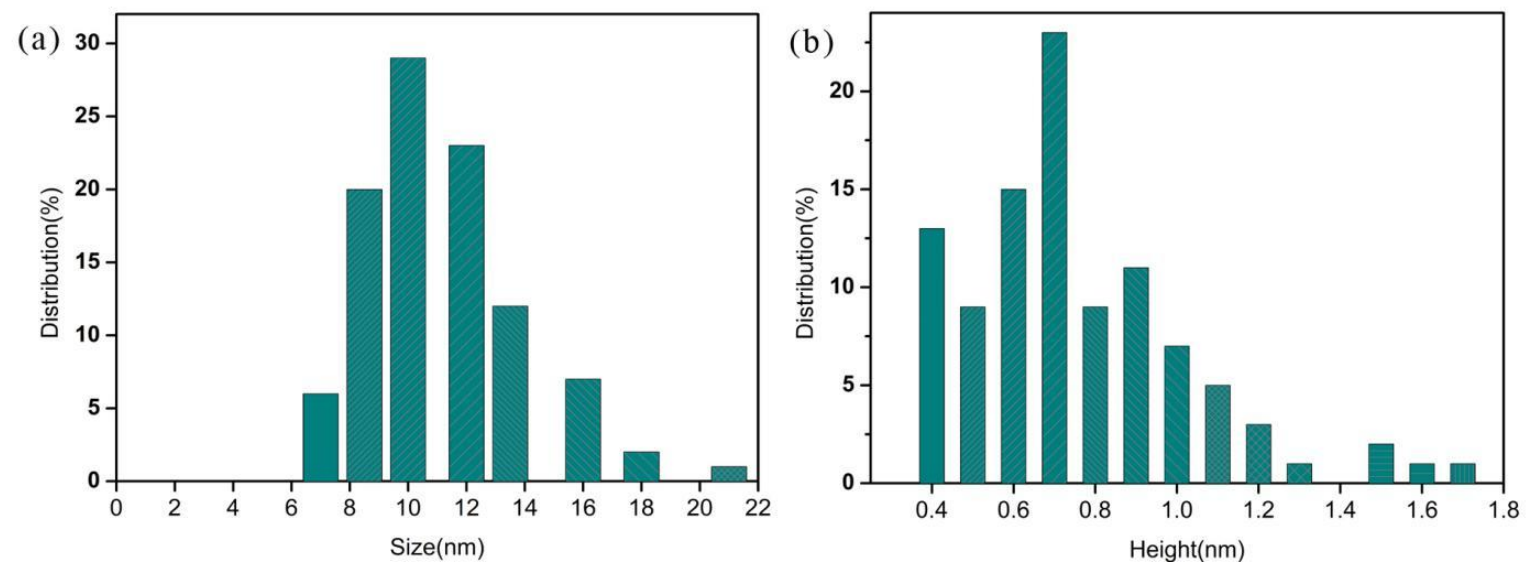

Figure2. (a) PSD by DLS. (b) The height of particles.

\section{RESULTS AND DISCUSSION}

The GQDs are sheet-like graphene fragments, so they can be regarded as elliptical structures. Pure water has highly insulated, GQDs are randomly distributed in pure water to form a two-phase conductive composite. With the GQDs volume fraction increase, isolated conductive particles form a continuous path. Graphene has good chemical stability, so GQDs solution is only a simple physical mixing and no chemical reaction.

\section{A Volume concentration dependence of the effective conductivity}

In this paper, the effective medium theory (EMT) model, the porous flow model, and the simplified GEM model are used to fit the experimental datum. The effective medium theory (EMT) model is not suitable for low concentration of composite materials, because the fitting result of the selfconsistent model in the low concentration phase is approximately constant. 
According to the average polarization theory and the literature [14], we can obtain that the percolation thresholds of graphene of polymer composites is less than 0.01 from this equation. The parameter $\alpha$ is critical to the porous flow theory model, the theoretical value of $\alpha$ is generally 1.65 2.0 in a three-dimensional conductive network, the parameter $\alpha$ is usually taken as $1-1.3$ in a two-dimensional conductive network. The parameter $\alpha$ is usually obtained through computer simulation. For the actual system, due to the influence of the morphology and the system dimension of the conductive particles, the actual value of $\alpha$ often exceeds the above theory.

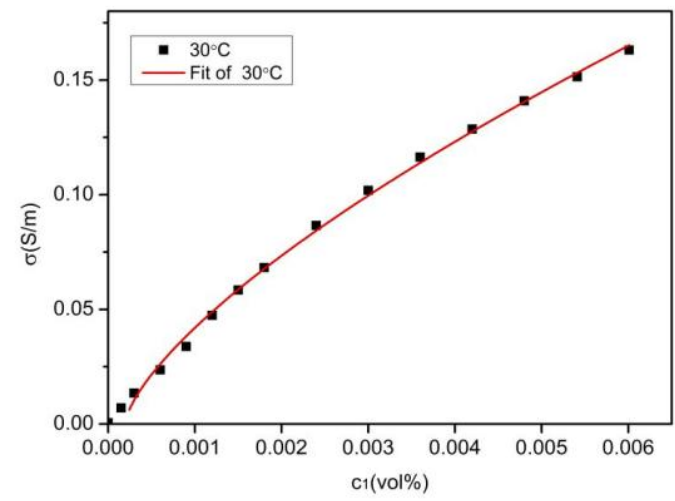

Figure3. Fitting based on porous flow theory $\left(\mathrm{t}=30^{\circ} \mathrm{C}\right)$.

Figure 3 shows the fitting curves of porous flow model based on the experimental datum. Because the minimum volume concentration of the experimental data was $1.5 \mathrm{E}-4$, so the percolation threshold $\kappa^{*}$ was set to $1 \mathrm{E}-4$, the percolation threshold $\kappa^{*}$ was $1.91 \mathrm{E}-4$, the conductivity of graphene quantum dots was $5.8 \mathrm{~S} / \mathrm{m}$ and the parameter $\alpha$ was 0.69 fitted from formula (2). The conductivity will increase rapidly when the volume fraction is near the percolation threshold. It can be seen that the percolation threshold is less than 1.5E-4 from the Fig.3. Therefore, the percolation thresholds estimated have little difference from the fit result and experimental datum.

As shown in Fig.4a, the simulated curve of the effective conductance of the GQDs solution by the GEM model. The percolation threshold $\kappa^{*}$ is still set as $1 \mathrm{E}-4$, the percolation

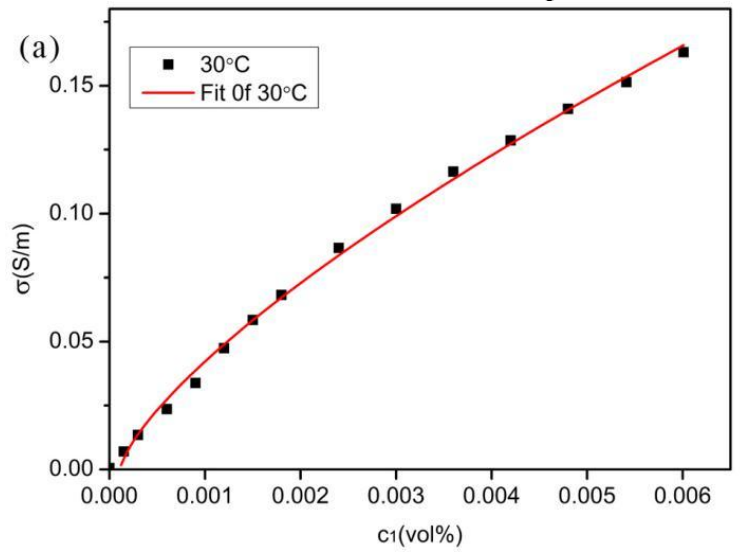

threshold $\kappa^{*}$ is $1.11 \mathrm{E}-4$, the conductivity of GQDs is 6.76 $\mathrm{S} / \mathrm{m}$ and the parameter $\alpha$ is 0.72 by fitting (4). The above analysis shows that the simulated datum and experimental datum consistent. The GEM model fit well at different temperatures in Fig. $4 \mathrm{~b}$.

\section{B Temperature dependence of the effective conductivity}

The base material of GQDs solution is pure water and it is insulation, so it belongs to the tunnel conductive system. Temperature has a great influence on the conductivity of composite materials. With the temperature increase, the ability of electrons cross the basement barrier is enhanced, the experimental datum be consistent with this law, however, the fitting degree of the tunnel conductivity theory to the experimental datum only 0.44 . The modified tunneling conductivity model has obtained combined with the tunneling and Arrhenius equations.

As shown in Fig.5a, the simulated image of the modified tunnel model for experimental datum. The value of activation energy Q of GQDs is 5.32E-20, and the value of parameter $\alpha$ is 1.53 obtained by the formula (10) simulation the simulation results are consistent with the experimental data. Fig.5b shows the applicability of the modified tunneling theory to different concentrations of graphene quantum dots solution.

\section{Magnetic field and frequency dependence of the effective conductivity}

From Fig.6, after application magnetic field the conductivity value has a little increase compare with no magnetic field. The differences can be ascribed to the magnetic field has little effect on the graphene quantum dots solution.

As is shown in Fig.6a, the conductivity of the graphene quantum dots solution increases at low frequency and decreases at high frequency. The effects of the magnetic field on the conductivity of GQDs solution are shown in Figs.6b. The conductivity shows increase at low frequency that can be attributed to the Debye-Falkenhagen effect. The conductivity decreases at high frequency, because the charged particles oscillate too fast at high frequency that the charged particles motion along a particular direction is smaller than that in low frequency.

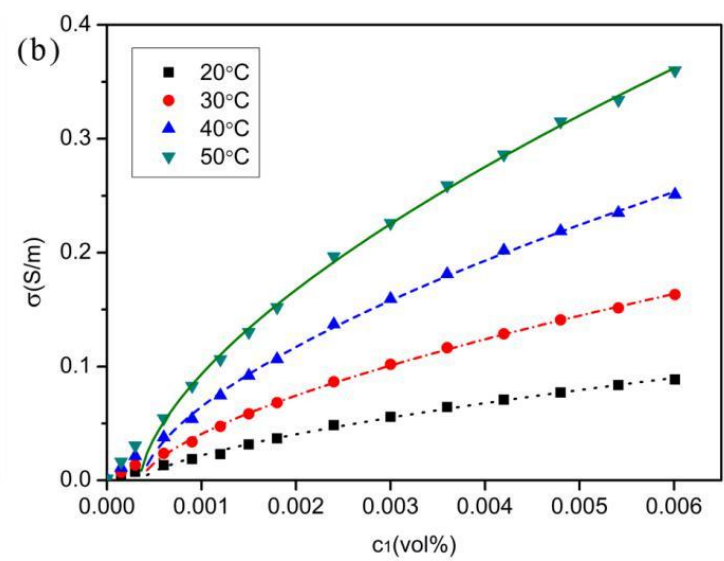

Figure4. (a) GEM model simulation. (b)The GEM model simulation at different temperatures. 

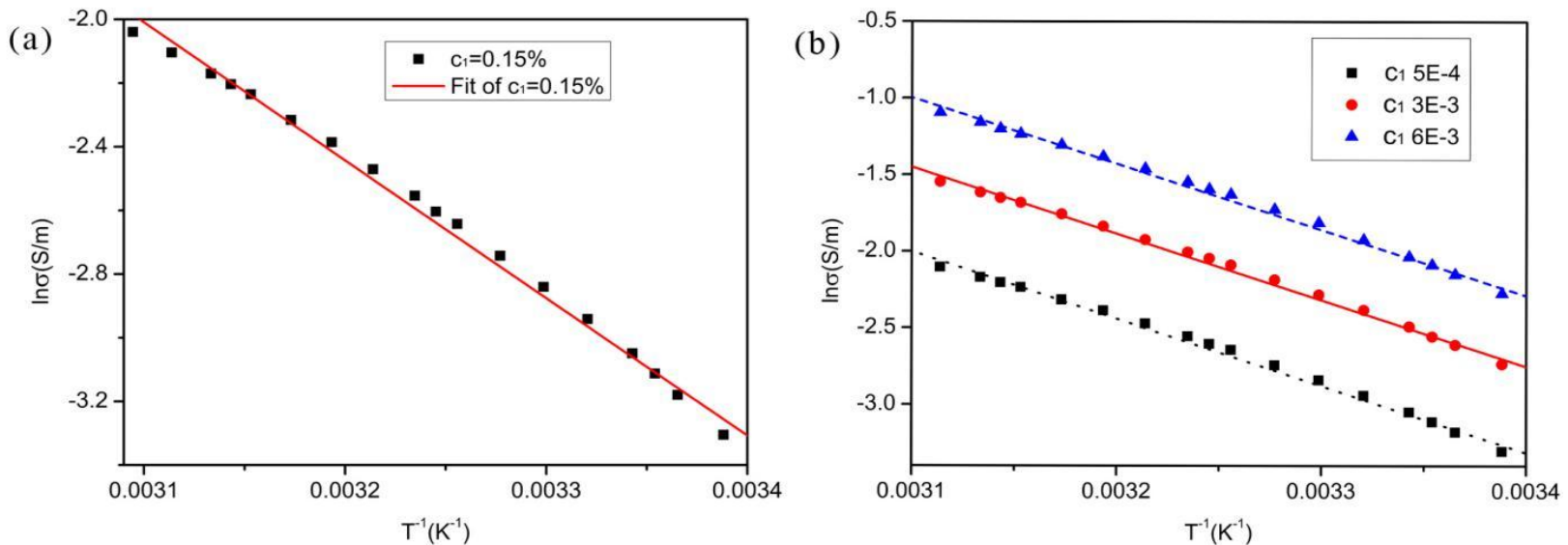

Figure5. Modified Tunnel Conduction Model Simulation(b)Modified tunnel model fitting at different concentrations.
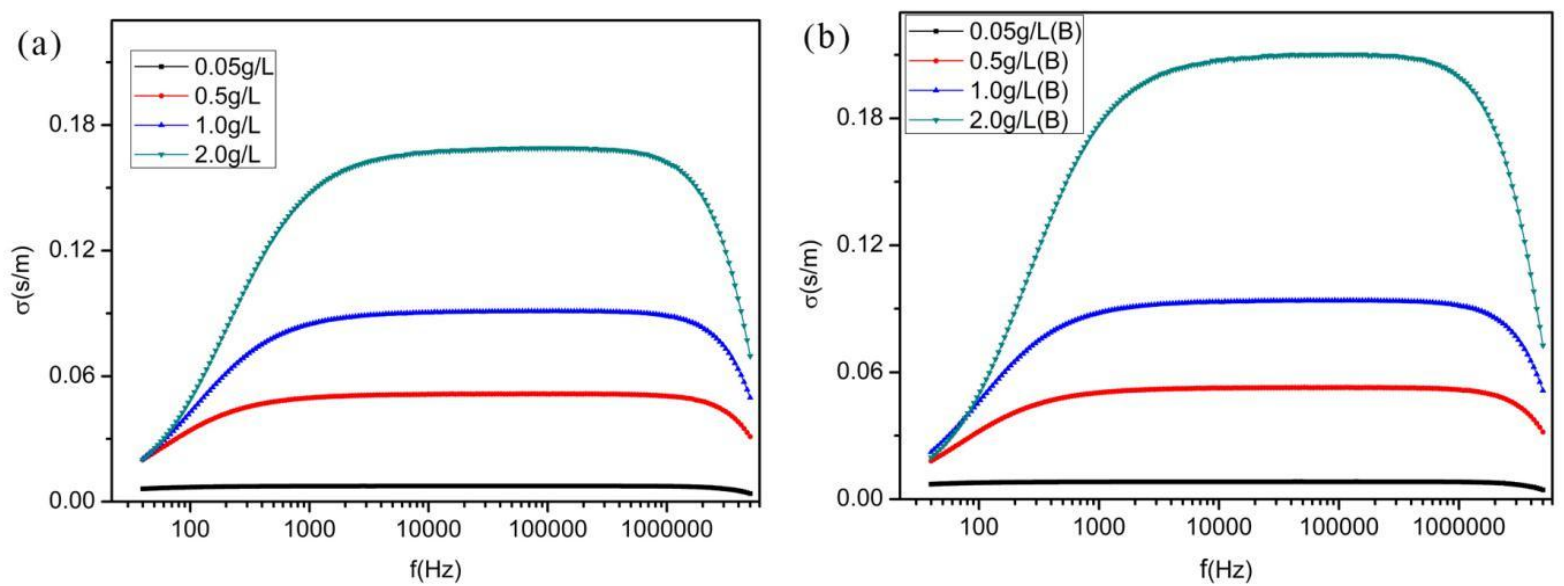

Figure6. Frequency dependence of conductivity of GDQs solution compare with under magnetic field. (c) without magnetic field (d)with magnetic field (at $\left.15^{\circ} \mathrm{C}\right)$.

\section{CONCLUSIONS}

The graphene quantum dots solution can be seen as a composite model. The trend of the fitting based on the effective medium theory (EMT) model is greatly deviated from the experimental data. The GEM model is based on the effective medium theory (EMT) model and porous flow theory model, can better simulate the effective conductivity change of the GQDs solution. The percolation threshold for the effective conductivity of the GQDs solution is affected by many factors. It not only related the conductive materials of the conductive properties, but also related the shape and size of the conductive material.

According to the tunnel theory model and Arrhenius equation, we can further modify the tunnel conductivity model. Therefore, the modified model fit the temperature dependence of the effective conductivity of GQDs solution better. However, the key index of the system in the model still needs a large number of experiments and further researches to determine.

In addition, we also measured the magnetic field and frequency dependence of the effective conductivity of the GQDs solution.

\section{ACKNOWLEDGMENTS}

The work was done under the guidance of professor Yufeng Peng. Part of funding was provided by Foundation of Henan Educational Committee (Grant No.18A510013). We thank the comments by the anonymous reviewer.

\section{REFERENCES}

[1] Liu W W, Feng Y Q, Yan X B, et al. Supercapacitors: Superior Micro - Supercapacitors Based on Graphene Quantum Dots (Adv. Funct. Mater. 33/2013). Advanced Functional Materials, 2013, 23(33):4111-4122.

[2] Gupta V, Chaudhary N, Srivastava R, et al. Luminscent graphene quantum dots for organic photovoltaic devices. Journal of the American Chemical Society, 2011, 133(26):9960.

[3] Duan J, Tang Q, Li R, et al. Multifunctional graphene incorporated polyacrylamide conducting gel electrolytes for efficient quasi-solidstate quantum dot-sensitized solar cells. Journal of Power Sources, 2015, 284:369-376.

[4] Ruiz V, Fernández I, Carrasco P, et al. Graphene quantum dots as a novel sensing material for low-cost resistive and fast-response humidity sensors. Sensors \& Actuators B Chemical, 2015, 218:73-77. 
[5] Kim S, Hwang S W, Kim M K, et al. Anomalous Behaviors of Visible Luminescence from Graphene Quantum Dots: Interplay between Size and Shape. Acs Nano, 2012, 6(9):8203-8.

[6] Roushani M, Mavaei M, Rajabi H R. Graphene quantum dots as novel and green nano-materials for the visible-light-driven photocatalytic degradation of cationic dye. Journal of Molecular Catalysis A Chemical, 2015, 409(2):102-109.

[7] Hasanzadeh M, Karimzadeh A, Sadeghi S, et al. Graphene quantum dot as an electrically conductive material toward low potential detection: a new platform for interface science. Journal of Materials Science Materials in Electronics, 2016, 27(6):6488-6495.

[8] Zhang W, Gan J. Synthesis of blue-photoluminescent graphene quantum dots/polystyrenic anion-exchange resin for $\mathrm{Fe}(\mathrm{III})$ detection. Applied Surface Science, 2016, 372:145-151.

[9] Zhao J, Zhao L, Lan C, et al. Graphene quantum dots as effective probes for label-free fluorescence detection of dopamine. Sensors \& Actuators B Chemical, 2016, 223:246-251.
[10] Shen J, Zhu Y, Yang X, et al. Graphene quantum dots: emergent nanolights for bioimaging, sensors, catalysis and photovoltaic devices. Cheminform, 2012, 43(29):3686.

[11] Li M, Wu W, Ren W, et al. Synthesis and upconversion luminescence of N-doped graphene quantum dots. Applied Physics Letters, 2012, 101(10):768.

[12] Chong Y, Ma Y, Shen $\mathrm{H}$, et al. The in vitro and in vivo toxicity of graphene quantum dots. Biomaterials, 2014, 35(19):5041.

[13] Schwaab M, Pinto J C. Optimum reference temperature for reparameterization of the Arrhenius equation. Part 1: Problems involving one kinetic constant. Chemical Engineering Science, 2007, 62(10):2750-2764.

[14] Torquato S, Hyun S. Effective -medium approximation for composite media.Realizable single-scale dispersions. Appl Phys, 2001;89(3):1725-1729. 